\title{
Endoscopic Ultrasound in the Diagnosis of Pancreatoduodenal Groove Pathology: Report of Three Cases and Brief Review of the Literature
}

\author{
Inés C. Oría, Juan E. Pizzala, Augusto M. Villaverde, Juan C. Spina, Analía V. Pasqua, Julio C. Lazarte, Oscar M. Mazza and \\ Mariano M. Marcolongo
}

Department of Gastroenterology, Hospital Italiano de Buenos Aires, Buenos Aires, Argentina

The pancreatoduodenal groove is a small area where pathologic processes involving the distal bile duct, duodenum, pancreatic head, ampulla of Vater, and retroperitoneum converge. Despite great advances in imaging techniques, a definitive preoperative diagnosis is challenging because of the complex anatomy of this area. Therefore, surgical intervention is frequently required because of the inability to completely exclude malignancy.

We report 3 cases of patients with different groove pathologies but similar clinical and imaging presentation, and show the essential role of endoscopic ultrasound (EUS) in making a specific preoperative diagnosis, excluding malignancy in the first case, changing diagnosis in the second case, and confirming malignancy in the third case. EUS was a fundamental tool in this cohort of patients, not only because of its ability to provide superior visualization of a difficult anatomical region, but because of the ability to guide precise, realtime procedures, such as fine-needle aspiration. Clin Endosc 2019;52:196-200

Key Words: Pancreatoduodenal groove; Endoscopic ultrasound; Fine needle aspiration; Pancreatic cancer; Groove pancreatitis

\section{INTRODUCTION}

The pancreatoduodenal groove is an anatomic space bordered by the head of the pancreas, duodenum, and common bile duct. This is an important landmark where pathologic processes that involve the pancreatic head, duodenum, distal pancreatobiliary tract, duodenal papilla, and retroperitoneum converge. ${ }^{1,2}$ Differential diagnosis includes a spectrum of entities, from anatomical variants with no clinical impact to malignancies with bad prognosis, such as pancreatic adenocarci-

Received: June 7, 2018 Revised: August 30, 2018

Accepted: August 31, 2018

Correspondence: Inés Cecilia Oría

Department of Gastroenterology, Hospital Italiano de Buenos Aires, Perón 4190, C1199ABB, Buenos Aires, Argentina

Tel: +54-91-1405-12713, Fax: +54-11-4959-0200, E-mail: Ines.oria@hospitalitaliano.org.ar

ORCID: https://orcid.org/0000-0001-8150-4670

(c) This is an Open Access article distributed under the terms of the Creative Commons Attribution Non-Commercial License (http://creativecommons.org/ licenses/by-nc/3.0) which permits unrestricted non-commercial use, distribution, and reproduction in any medium, provided the original work is properly cited. noma. This area poses a unique diagnostic challenge because of the complex anatomy of the region, sometimes making it difficult to perform a preoperative differential diagnosis, with the possible serious consequences of an incorrect diagnosis. ${ }^{3.5}$ Endoscopic ultrasound (EUS) is currently the tool of choice to evaluate this area, due to its accessibility, accuracy, and the ability to perform endoscopic fine-needle aspiration (FNA). ${ }^{6}$

The aim of this article was to report three patients with similar clinical and radiological presentations and o show the essential role of EUS in making a preoperative diagnosis.

\section{CASE REPORTS}

\section{Case 1}

A 61-year-old male smoker and alcoholic was referred to our center for diagnosis and treatment of a pancreatic mass. He had consulted at his local hospital for a 30-pound weight loss and epigastric pain radiating to the back for 3 months. Laboratory tests and tumor markers were within normal lim- 
its. Magnetic resonance cholangiopancreatography showed a poorly defined mass of $25 \times 21 \times 41 \mathrm{~mm}$ compromising the pancreatic head and pancreatoduodenal groove, with cystic areas and thickening of the first and second duodenal portions. A normal pancreatic body and tail were observed, with a non-dilated pancreatic duct and no compression of the bile duct, which was slightly deformed (Fig. 1). EUS showed diffuse chronic pancreatitis with focal hypoechogenicity and nodularity in the head, but without compromise of the intrapancreatic bile duct or pancreatic duct. The duodenal wall was thickened by a hypoechoic formation compromising the pancreatic parenchyma, and an anechoic lesion of $15 \mathrm{~mm}$ was compatible with an intraparietal duodenal cyst. FNA was performed on the focal area for histopathological examination, and the cyst for physical-chemical and cytological analysis. Endoscopically, edematous mucosa with a polypoid appearance and narrowing of the second part of the duodenum was observed, and biopsies were performed (Fig. 2). Histopathological examination of the focal mass revealed acini in the context of an acute inflammatory infiltrate, and accumulation of fusiform cells without atypical epithelial cells. Vimentin and synaptophysin were expressed but pan-cytokeratin AE1AE3 was not detected in spindle cells. The duodenum showed chronic bulboduodenitis and foveolar metaplasia without dysplasia; cyst analysis revealed a CEA of $<0.5 \mathrm{ng} / \mathrm{mL}$ and negative cytology. With the diagnosis of chronic pancreatitis and acute compromise of the gastroduodenal groove, pancreatic enzymes and elimination of risk factors were indicated with good clinical response, weight gain, and normalization of imaging findings 6 months later.

\section{Case 2}

A 72-year-old female with a history of breast cancer treated with surgery and radiotherapy 2 years before, consulted for significant weight loss, anorexia, epigastric pain radiating to the back, and occasional vomiting for 3 months. Physical examination and routine blood tests were unremarkable. Computed tomography (CT) showed a non-dilated biliary tract, atrophic pancreatic gland with globular appearance of the head and uncinate process and calcifications, and concentric parietal thickening at the level of the second part of the duodenum. The surrounding fat was altered, especially in the groove area, and was associated with a fine fluid band (Fig. $3 \mathrm{~A}, \mathrm{~B})$. Although these findings could correspond to groove pancreatitis, another origin could not be ruled out; EUS was performed, and showed a pancreas with normal echogenicity without focal lesions. At the level of the first and second part of the duodenum, EUS revealed a semi-circumferentially-thickened duodenal wall of up to $11 \mathrm{~mm}$ with disruption of the layered configuration. Endoscopically, a large ulcer
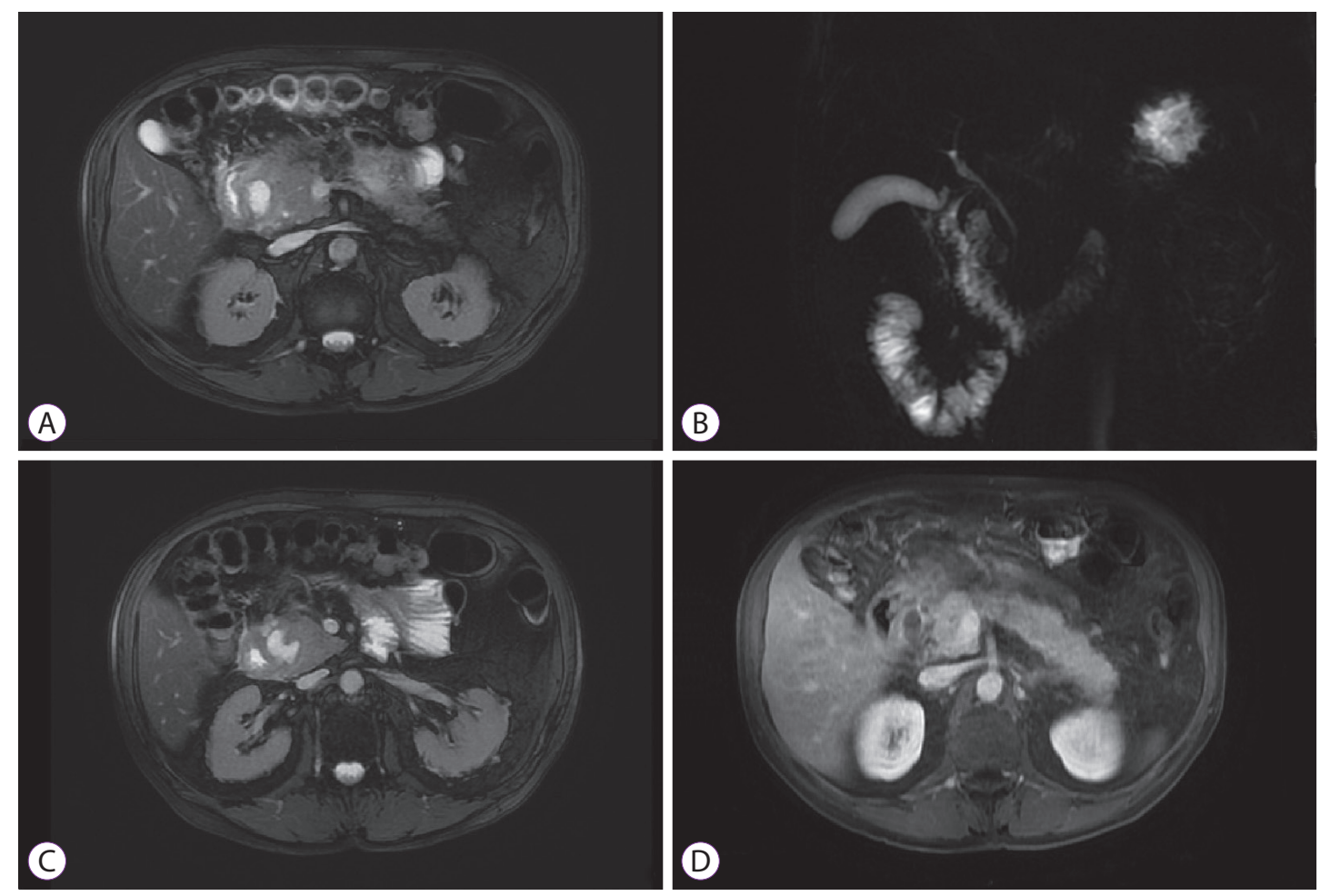

Fig. 1. (A-D) Magnetic resonance cholangiopancreatography. Cephalic pancreatic mass with poorly defined margins and heterogeneous fluid signal, without significant contrast enhancement. The distal bile duct is slightly deformed without compression. Non-dilated pancreatic duct. Thickening of the second part of duodenum. Cystic lesion at the pancreaticoduodenal groove. 
occupying half of the duodenal circumference covered with fibrin was observed at the duodenal bulb. The surrounding mucosa was edematous and congested, with an inflammatory appearance (Fig. 3C, D). Histopathological examination of the ulcer revealed mucosa with edema and reactive changes, but no atypical cells. High-dose omeprazole was prescribed for 8 weeks with good clinical response and normalization of radiological and endoscopic images after 2 months.

\section{Case 3}

A 72-year-old male smoker was referred to our center with chronic epigastric pain, significant weight loss for 3 months, and obstructive jaundice. Laboratory tests showed elevation of pancreatic and hepatic enzymes: amylase $220 \mathrm{IU} / \mathrm{L}$ (normal value [NV] 25-125 IU/L), gamma glutamyl transpeptidase $630 \mathrm{IU} / \mathrm{L}$ (NV 9-55 IU/L), alkaline phosphatase $242 \mathrm{IU} / \mathrm{L}$ (NV 31-100 IU/L), alanine and aspartate aminotransferase (ALT/ AST 249/148 IU/L [NV 10-40/10-42 IU/L]), total bilirubin $15.4 \mathrm{mg} / \mathrm{dL}$ (NV $0.5 \mathrm{mg} / \mathrm{dL}$ ), direct bilirubin $8 \mathrm{mg} / \mathrm{dL}$ (NV 0-0.4 mg/dL), and elevated carbohydrate antigen (CA) 19-9 $(870 \mathrm{U} / \mathrm{mL}$ [NV $30 \mathrm{U} / \mathrm{mL}])$. CT showed biliary tract dilation without identification of the terminal portion, where a change in caliber was noted with wall enhancement. Thickening of the second part of the duodenum wall was observed, with 3 intraparietal cystic images and a hypodense tissue inter- posed between the pancreas and duodenum. The pancreas was slightly diminished in size, with adequate enhancement with intravenous contrast. The findings were compatible with cystic dystrophy of the duodenal wall, and dilation of the bile duct was probably secondary to the presence of an inflammatory process present at the level of the papilla that compromised the terminal bile duct (Fig. 4A, B). Even though the imaging work-up suggested duodenal dystrophy, we had a high suspicion of malignancy; EUS-FNA showed a $20 \times 15$ $\mathrm{mm}$ hypoechoic heterogeneous cephalic mass compromising the duodenal wall, which was thickened by the presence of 3 intraparietal cysts. The bile duct was dilated $(15 \mathrm{~mm})$ and the pancreatic duct was also slightly dilated (Fig. 4C, D). FNA of the lesion was performed, revealing atypical tumor proliferation and cells with variable degrees of anisokaryosis in an inflammatory environment. Pancreaticoduodenectomy was performed (Fig. 4E, F) and anatomic-pathologic evaluation revealed pancreatic adenocarcinoma with infiltration of the duodenal submucosa and tumor-free surgical margins (T3N1-M0).

\section{DISCUSSION}

Abnormalities of the pancreatoduodenal groove can be
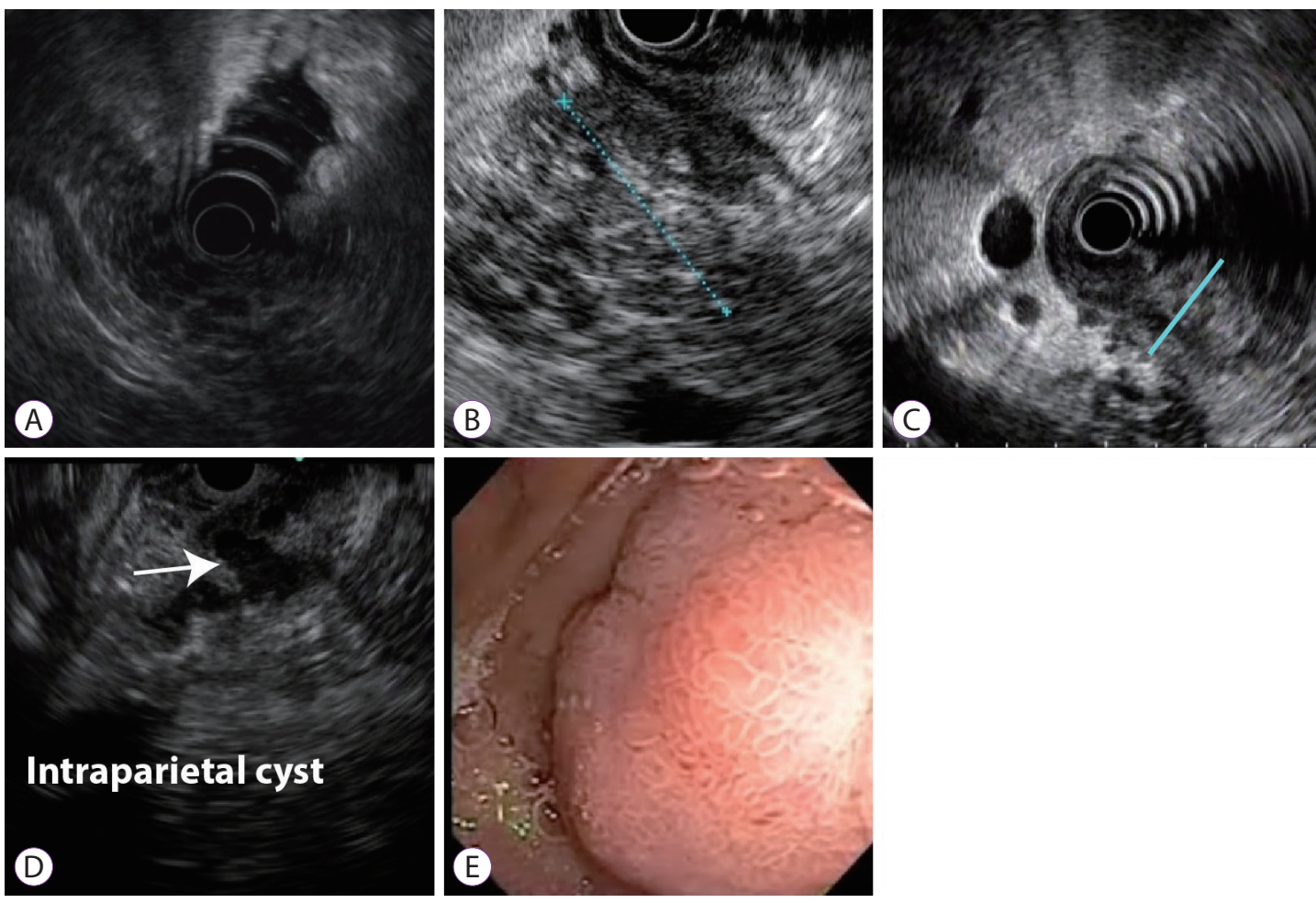

Fig. 2. Endoscopic ultrasound with fine-needle aspiration. (A) Pancreatic body and tail: lobularity with honeycombing, hyperechoic foci without shadowing. (B) Pancreatic head: hypoechoic mass with irregular margins, hyperechoic foci and lobularity. (C) Semi-circumferential parietal thickening of the second part of the duodenum. (D) Intraparietal duodenal cyst. (E) Endoscopically, edematous mucosa with a polypoid hyperplastic appearance in the second part of the duodenum. 

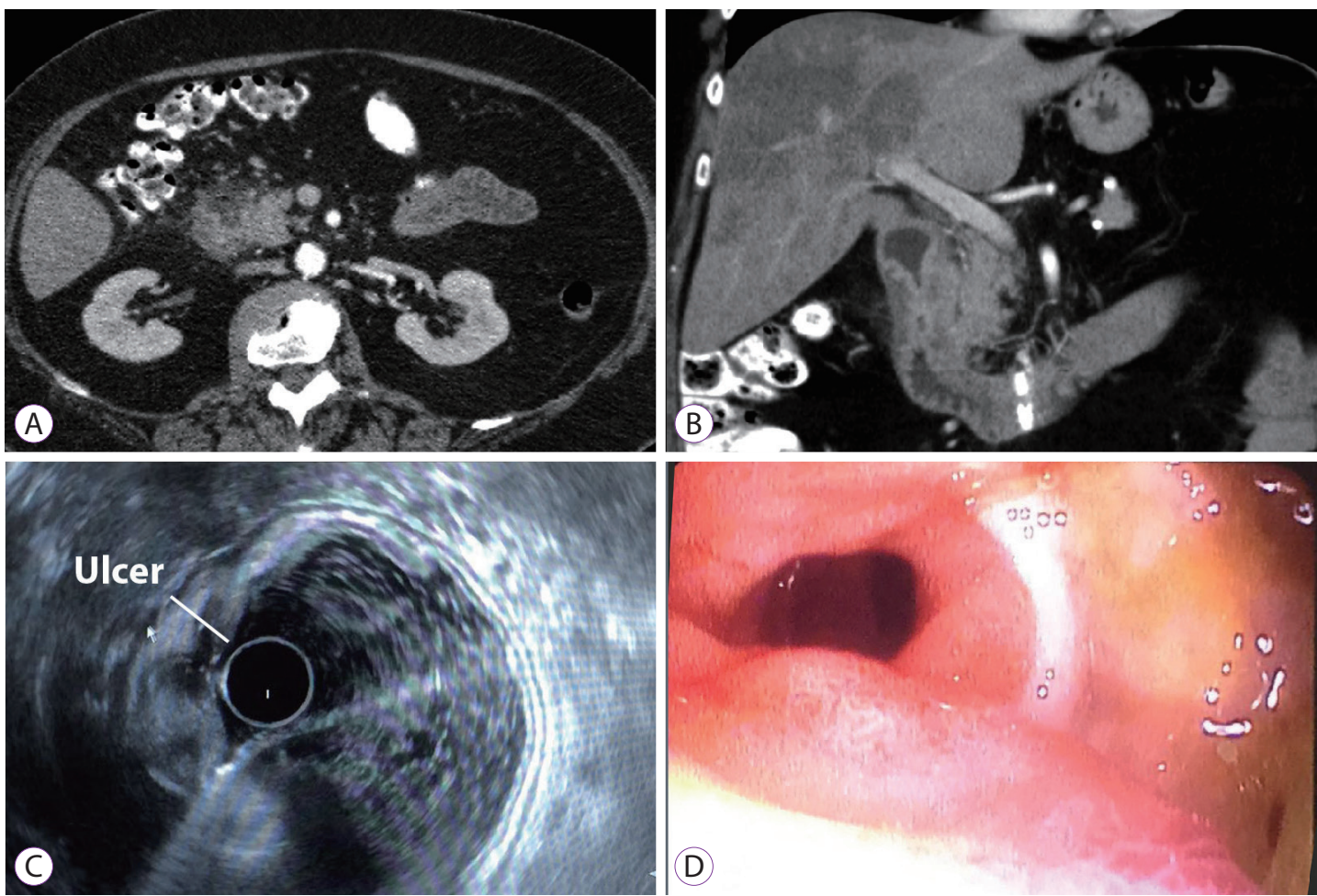

Fig. 3. (A, B) Multiple detector computed tomography, axial portal venous phase (A) and coronal portal venous phase (B), showing globular appearance of pancreatic head and uncinate process. Concentric duodenal wall thickening with a diverticular image appearance on the anterior wall associated with periduodenal fat stranding and an air bubble. Note also a fine fluid band. (C) Endoscopic ultrasound (EUS). Normal echogenicity of the pancreatic gland without focal lesions. At the level of first and second part of the duodenum, EUS revealed a semi-circumferentially-thickened duodenal wall with disruption of layer configuration. (D) Endoscopically, at the duodenal knee, a large ulcer occupying half of the duodenal circumference covered with fibrin was seen.
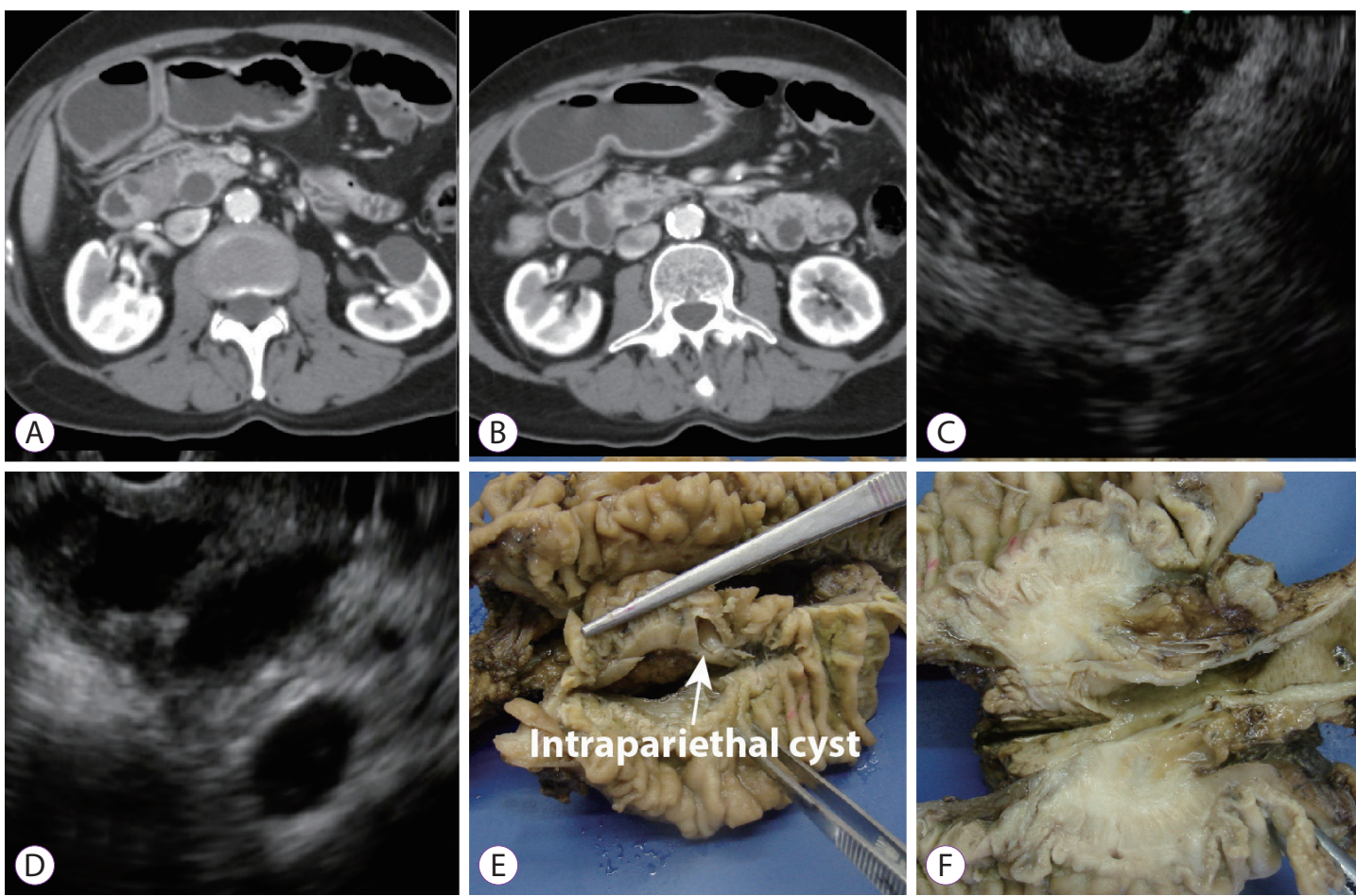

Fig. 4. (A, B) Computed tomography: Axial plane images showed a hypodense lesion in the groove area with paraduodenal cysts and a dilated biliary tree. (C) Endoscopic ultrasound (EUS). Pancreatic head: Hypoechoic mass with irregular margins, slightly dilated pancreatic duct. (D) EUS. Parietal thickening of the second part of the duodenum. Intraparietal duodenal cysts. (E, F) Pathological specimen: a pale and indurated lesion located in the pancreatic head with cyst formation on the duodenal wall. 
divided into 3 categories: (1) normal variants and congenital anomalies, such as pancreas divisum, santorinicele, annular pancreas, duodenal duplication cyst, and choledochal cysts; (2) acquired non-tumor abnormalities (traumatic, iatrogenic, inflammatory): such as duodenal hematoma, perforation and ulcers, groove pancreatitis, and pseudoaneurysms of the pancreatoduodenal artery; and (3) tumors: cephalic pancreatic adenocarcinoma and other periampullary tumors (neuroendocrine tumors, duodenal adenocarcinoma). ${ }^{2}$ The complex anatomic relationships of the structures in this small area have given rise to diagnostic challenges in which a variety of benign processes often mimic primary neoplasia of the involved structures. Unfortunately, despite advances in technology and the exhaustive use of imaging techniques, in many cases the diagnosis is made after surgical resection. Thus, the main challenge in this area is preoperative differentiation between benign and malignant disease, especially pancreatic adenocarcinoma. $^{5,7}$

EUS combines a high-frequency ultrasound probe with an endoscope; EUS is well established for imaging the gastrointestinal (GI) tract by allowing visualization of the lumen and wall layers. It overcomes limitations of transabdominal ultrasound such as abdominal girth and overlying gas. As a result of the probe being positioned in close proximity to the pancreas and extrahepatic biliary system, this tool allows extraordinary high-resolution images with subtle anatomic detail, providing a level of analysis not routinely possible with either CT or magnetic resonance imaging. Moreover, it has the ability to allow targeted biopsies of the abdominal viscera immediately adjacent to the GI tract. Therefore, EUS has a leading role in the evaluation of the pancreatobiliary region, especially the pancreatoduodenal groove. ${ }^{8-10}$

In the 3 cases reported, our presumptive diagnosis was inflammation in the pancreatoduodenal groove. Despite having high-quality images, EUS with or without FNA allowed us to make a correct preoperative diagnosis of pathology, which was different in the 3 cases.

In the first case, the patient was a heavy drinker and had a history of smoking so it was reasonable to suspect chronic pancreatitis; however, ruling out a malignancy was mandatory due to the relationship between pancreatic cancer and smoking and the worse prognosis of this malignancy. The clinical and radiological improvement after a change in habits was remarkable in this patient.

The second case showed important differences between the pancreatic morphology observed with CT and EUS; while CT showed an enlarged pancreatic head, EUS showed an absolutely normal pancreatic parenchyma, and the symptoms and signs in this patient were all attributed to a large duodenal ulcer whose presentation was uncommon.

In the last case, EUS allowed planning of surgery on the basis of a firm diagnosis. Although clinical presentation of duodenal dystrophy and pancreatic cancer may overlap, weight loss and CA 19-9 were significant in this patient, and the diagnosis of pancreatic adenocarcinoma was always kept in mind.

To our knowledge, the literature on the role of EUS in the differential diagnosis of pancreatoduodenal groove pathology is limited, and even in the most specialized centers, many gastroenterologists, surgeons, and radiologists are not familiar with this anatomical region. ${ }^{8-10}$

In conclusion, we consider EUS to be a valuable tool in the diagnostic work-up of patients with pancreatoduodenal groove pathology, and recommend that it be used early in evaluation.

\section{Conflicts of Interest}

The authors have no financial conflicts of interest.

\section{Author Contributions}

Investigation: Augusto M. Villaverde, Julio C. Lazarte

Supervision: Oscar M. Mazza, Mariano M. Marcolongo

Visualization: Juan C. Spina

Writing-original draft: Inés C. Oría, Juan E. Pizzala, Analía V. Pasqua

\section{REFERENCES}

1. Yu J, Fulcher AS, Turner MA, Halvorsen RA. Normal anatomy and disease processes of the pancreatoduodenal groove: imaging features. AJR Am J Roentgenol 2004;183:839-846.

2. Hernandez-Jover D, Pernas JC, Gonzalez-Ceballos S, Lupu I, Monill JM, Pérez C. Pancreatoduodenal junction: review of anatomy and pathologic conditions. J Gastrointest Surg 2011;15:1269-1281.

3. DeSouza K, Nodit L. Groove pancreatitis: a brief review of a diagnostic challenge. Arch Pathol Lab Med 2015;139:417-421.

4. Raman SP, Salaria SN, Hruban RH, Fishman EK. Groove pancreatitis: spectrum of imaging findings and radiology-pathology correlation. AJR Am J Roentgenol 2013;201:W29-W39.

5. Goransky J, Alvarez FA, Picco P, Spina JC, Santibañes M, Mazza O. Groove pancreatitis vs groove pancreatic adenocarcinoma. Report of two cases and review of the literature. Acta Gastroenterol Latinoam 2013;43:248-253.

6. Fusaroli P, Kypraios D, Caletti G, Eloubeidi MA. Pancreatico-biliary endoscopic ultrasound: a systematic review of the levels of evidence, performance and outcomes. World J Gastroenterol 2012;18:4243-4256.

7. Ray S, Ghatak S, Misra D, et al. Groove pancreatitis: report of three cases with brief review of literature. Indian J Surg 2017;79:344-348.

8. Vilmann P, Jacobsen GK, Henriksen FW, Hancke S. Endoscopic ultrasonography with guided fine needle aspiration biopsy in pancreatic disease. Gastrointest Endosc 1992;38:172-173.

9. Shetty D, Bhatnagar G, Sidhu HS, Fox BM, Dodds NI. The increasing role of endoscopic ultrasound (EUS) in the management of pancreatic and biliary disease. Clin Radiol 2013;68:323-335.

10. ASGE Standards of Practice Committee, Gan SI, Rajan E, et al. Role of EUS. Gastrointest Endosc 2007;66:425-434. 